ARTICLE

\title{
Cryo-EM structure of mycobacterial cytochrome $b d$ reveals two oxygen access channels
}

Weiwei Wang 1,2,3,4,8, Yan Gao (1) 1,8, Yanting Tang ${ }^{2}$, Xiaoting Zhou 1,3,4, Yuezheng Lai ${ }^{2}$, Shan Zhou ${ }^{2}$,

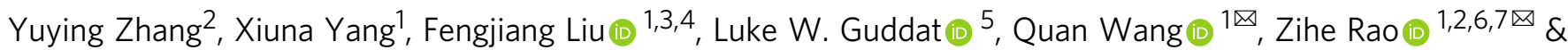
Hongri Gong (1) ${ }^{2 凶}$

Cytochromes bd are ubiquitous amongst prokaryotes including many human-pathogenic bacteria. Such complexes are targets for the development of antimicrobial drugs. However, an understanding of the relationship between the structure and functional mechanisms of these oxidases is incomplete. Here, we have determined the $2.8 \AA$ structure of Mycobacterium smegmatis cytochrome $b d$ by single-particle cryo-electron microscopy. This $b d$ oxidase consists of two subunits $\mathrm{CydA}$ and $\mathrm{CydB}$, that adopt a pseudo two-fold symmetrical arrangement. The structural topology of its Q-loop domain, whose function is to bind the substrate, quinol, is significantly different compared to the C-terminal region reported for cytochromes bd from Geobacillus thermodenitrificans (G. th) and Escherichia coli (E. coli). In addition, we have identified two potential oxygen access channels in the structure and shown that similar tunnels also exist in $G$. th and $E$. coli cytochromes $b d$. This study provides insights to develop a framework for the rational design of antituberculosis compounds that block the oxygen access channels of this oxidase.

\footnotetext{
${ }^{1}$ Shanghai Institute for Advanced Immunochemical Studies and School of Life Science and Technology, ShanghaiTech University, Shanghai, China. ${ }^{2}$ State Key Laboratory of Medicinal Chemical Biology, Frontiers Science Center for Cell Responses, College of Life Sciences, Nankai University, Tianjin, China. ${ }^{3}$ CAS Center for Excellence in Molecular Cell Science, Shanghai Institute of Biochemistry and Cell Biology, Chinese Academy of Sciences, Shanghai, China.

${ }^{4}$ University of Chinese Academy of Sciences, Beijing, China. ${ }^{5}$ School of Chemistry and Molecular Biosciences, The University of Queensland, Brisbane, QLD, Australia. ${ }^{6}$ National Laboratory of Biomacromolecules, CAS Center for Excellence in Biomacromolecules, Institute of Biophysics, CAS, Beijing, China.

${ }^{7}$ Laboratory of Structural Biology, Tsinghua University, Beijing, China. ${ }^{8}$ These authors contributed equally: Weiwei Wang, Yan Gao.

凶email: wangq@shanghaitech.edu.cn; raozh@tsinghua.edu.cn; gonghr@nankai.edu.cn
} 
$\mathrm{R}$ espiratory oxygen reductases (terminal oxidases) comprise a series of structurally distinct enzymes that are widely distributed across all kingdoms of life. The heme-copper oxidases (HCO) and $b d$-type oxidases (cytochromes $b d$ ) are two well-known types of membrane-integrated terminal oxidases ${ }^{1,2}$. They catalyze the reduction of molecular oxygen $\left(\mathrm{O}_{2}\right)$ to water by the respiratory substrate, cytochrome $c$ or quinol, coupled to the generation of a proton motive force utilized for adenosine triphosphate (ATP) synthesis ${ }^{3,4}$. Compared to the well-characterized HCOs, cytochromes $b d$ have not been widely studied. These cytochromes are only present in prokaryotes, which include many human pathogens, and thus belong to an evolutionarily distinct oxidase family ${ }^{4}$.

Cytochrome bd oxidases possess a high affinity for oxygen ${ }^{5,6}$, which facilitates bacterial survival under $\mathrm{O}_{2}$-poor environments ${ }^{7,8}$. Apart from this, cytochromes $b d$ also endow bacteria with a number of vitally important physiological functions including enhancing tolerance to nitrosative stress ${ }^{9}$, contribute to resistance to hydrogen peroxide $^{10}$, suppress extracellular superoxide production ${ }^{11}$, and confer the ability to defend against antibacterial agents ${ }^{12}$. It is likely that these properties of cytochromes $b d$ promote virulence in a number of bacterial pathogens that cause serious infectious diseases to humans, such as Mycobacterium tuberculosis ${ }^{13}$, Brucella abortus ${ }^{14}$, as well as Salmonella Typhimurium ${ }^{15,16}$, Bacteroides ${ }^{7}$, and Listeria monocytogenes ${ }^{17}$. Since cytochrome $b d$ is a key enzyme for the survival of prokaryotes and is absent in mammals, it is a promising therapeutic target for the development of antibacterial agents ${ }^{4,18}$.

To date, two structures of the $b d$ oxidases have been reported. One is from Geobacillus thermodenitrificans ( $G$. th) ${ }^{19}$ and the other is from Escherichia coli $(E \text {. coli })^{20,21}$. These enzymes possess two key subunits CydA and CydB but vary in the numbers of additional small subunits that are associated with them. G. th $b d$ oxidase contains an association subunit called CydS and E. coli bd oxidase includes two association subunits named CydX and CydH (or CydY). Common features in these complexes are two $b$-type hemes (low-spin heme $b_{558}$ and high-spin heme $b_{595}$ ) and one $d$-type heme which are arranged in a triangular manner but with different relative positions in the two structures. These observations suggest that homologous $b d$ oxidases share a similar architecture but can vary in mechanistic detail ${ }^{21-24}$. Therefore, more structural information is needed to obtain a comprehensive knowledge of the structure and function of $b d$ oxidases. The properties of several mycobacterial cytochromes $b d$ have been investigated $^{12,18,24,25}$, but given they share only a low sequence identity (the lowest similarity score is $20 \%$ ) with the two previously reported cytochrome $b d$ structures it is difficult to rationalize the function of these cytochromes $b d^{18}$. In the present study, we have determined a $2.8 \AA$ cryo-electron microscopy (cryo-EM) structure of a dimeric $b d$ oxidase from Mycobacterium smegmatis $(\mathrm{Msm})$. In so doing, we have identified two potential oxygen access channels, which could be excellent targets for antituberculosis drug discovery.

\section{Results and discussion}

Overall structure of Mycobacterium smegmatis bd oxidase. $M s m$ bd oxidase was recombinantly expressed and purified to homogeneity (Supplementary Fig. 1a-d). The purified Msm bd enzyme is a stable and functional assembly with a turnover number of $21.6 \pm 2.8 \mathrm{e}^{-} \mathrm{s}^{-1}$ (Supplementary Fig. 1e). A $2.8 \AA$ resolution structure was determined in lipid nanodiscs using cryo-EM. Details for data collection and model statistics are provided (Supplementary Fig. 2 and Supplementary Table 1). Although the construct design, expression, and purification of $M s m$ bd oxidase were performed according to a procedure described previously for $G$. $t h^{19}$ and $E$. coli ${ }^{20,21} b d$ oxidases, only
CydA and CydB were observed. No additional small subunit similar to CydS/X or CydH/Y found in the G. $t h^{19}$ and E. coli ${ }^{20,21}$ $b d$ oxidases were observed in the Msm complex, noting that in the $G$. th and $E$. coli studies, the genes coding for the associated subunits CydS/X of G. $t h^{19} b d$ oxidase and CydH/Y of E. coli20,21 $b d$ oxidase were not included in the corresponding expression plasmid. Nonetheless, these two small subunits were observed to co-elute upon purification in both complexes. Thus, we believe that the Msm bd oxidase contains only two core subunits, CydA and CydB (Fig. 1a, b, and Supplementary Figs. 3, 4). This hypothesis is further supported by a BLAST search of the mycobacterial genomes which did not identify any subunits homologous to CydS/X and CydH/Y (Supplementary Fig. 5). In addition, $M s m$ bd oxidase in the absence of associated subunits is active, whereas the $E$. coli $b d$ oxidase is not active in the absence of its associated subunits ${ }^{26}$. In summary, mycobacterial $b d$ oxidase appears to only consist of the two core subunits and no other units. Notably, there is a high structural similarity between the $G$. th and E. coli counterparts compared to Msm core subunits, except for a little difference in the topology of CydB subunits between Msm and E. coli (Supplementary Fig. 6).

In terms of secondary structure, Msm bd oxidase, CydA, and CydB possess a common fold each consisting of nine transmembrane helices (TMHs). These two subunits are related by an approximate two-fold rotational axis of symmetry. The TMH domains can be divided into two four-helix bundles and one additional peripheral helix (Fig. 1c). The domains of the two subunits superpose well with a root mean square deviation of 3.6 $\AA$ for all the $\mathrm{Ca}$ atoms (Fig. 1c) and thus suggest that CydA and $\mathrm{CydB}$ evolved by a gene duplication event. The three heme groups $\left(b_{558}, b_{595}\right.$, and $\left.d\right)$ are clearly visible in the CydA subunit (Fig. $1 \mathrm{~b}$ and Supplementary Fig. 3). Given the two other $b d$ oxidases have associated subunits in their structures, our structure suggests that their presence is species-dependent.

Q-loop of $\boldsymbol{M}$. smegmatis $\boldsymbol{b} \boldsymbol{d}$ oxidase. The $b d$ oxidases are divided into the $S$ (short)- and the $\mathrm{L}$ (long)-subfamilies ${ }^{4}$. This is according to the length of the region of polypeptide referred to as the Q-loop (quinol-binding domain). Mycobacterial cytochromes $b d$ belong to the S-subfamily ${ }^{20}$. The Q-loop of CydA has a waterexposed domain (segment 257-339), connecting the 6 and 7 in the hydrophilic extracellular space ${ }^{19-21}$. It has two parts, one associated with the $\mathrm{N}$-terminal domain $\left(\mathrm{Q}_{\mathrm{N}}\right)$ and the other with the C-terminal domain $\left(\mathrm{Q}_{\mathrm{C}}\right)$. The $\mathrm{Q}_{\mathrm{N}}$-loop plays a functional role in the binding and oxidation of the quinol ${ }^{27,28}$ and the $\mathrm{Q}_{\mathrm{C}}$-loop is needed for the assembly/stability of the enzyme $22,23,25$.

In the present study, the densities corresponding to the Q-loop without and with aurachin D (a quinone analog inhibiting mycobacterial cytochrome $b d$ ) bound are not completely resolved (Supplementary Figs. 2 and 7). A comparison of these two structures does not show any conformational changes which may be a function of the resolution of the data or the fact that the region around the aurachin $\mathrm{D}$ is inherently disordered. Aurachin $\mathrm{D}$ is bound to the $\mathrm{Q}_{\mathrm{N}}-$ loop $^{20}$, potentially stabilizing the Q-loop, and has the ability to inhibit the activity of Msm cytochrome $b d^{29}$. The structure of the $\mathrm{Q}_{\mathrm{N}}$-loop in the E. coli enzyme is also not fully resolved ${ }^{20,21}$. Therefore, these structural data suggest that the $\mathrm{Q}_{\mathrm{N}^{-}}$ loop is intrinsically flexible. Its flexibility may be required for the rapid binding and release of the quinols. The remaining segments of the Q-loop in the Msm bd oxidase structure are well resolved. At the periplasmic side of $\mathrm{TMH} \mathrm{6,} \mathrm{there} \mathrm{is} \mathrm{a} \mathrm{short} \mathrm{horizontal}$ helix, Qh1, that includes the highly conserved residues Lys ${ }^{260}$ and $\mathrm{Glu}^{265}$ (Lys $^{252}$ and Glu ${ }^{257}$ in G. th and E. coli) ${ }^{19-21}$, critical for quinol substrate binding and electron $\operatorname{transfer}^{28}$ (Fig. 2a). The structure of $Q h 1$ is conserved with respect to the $G$. th and E. coli 

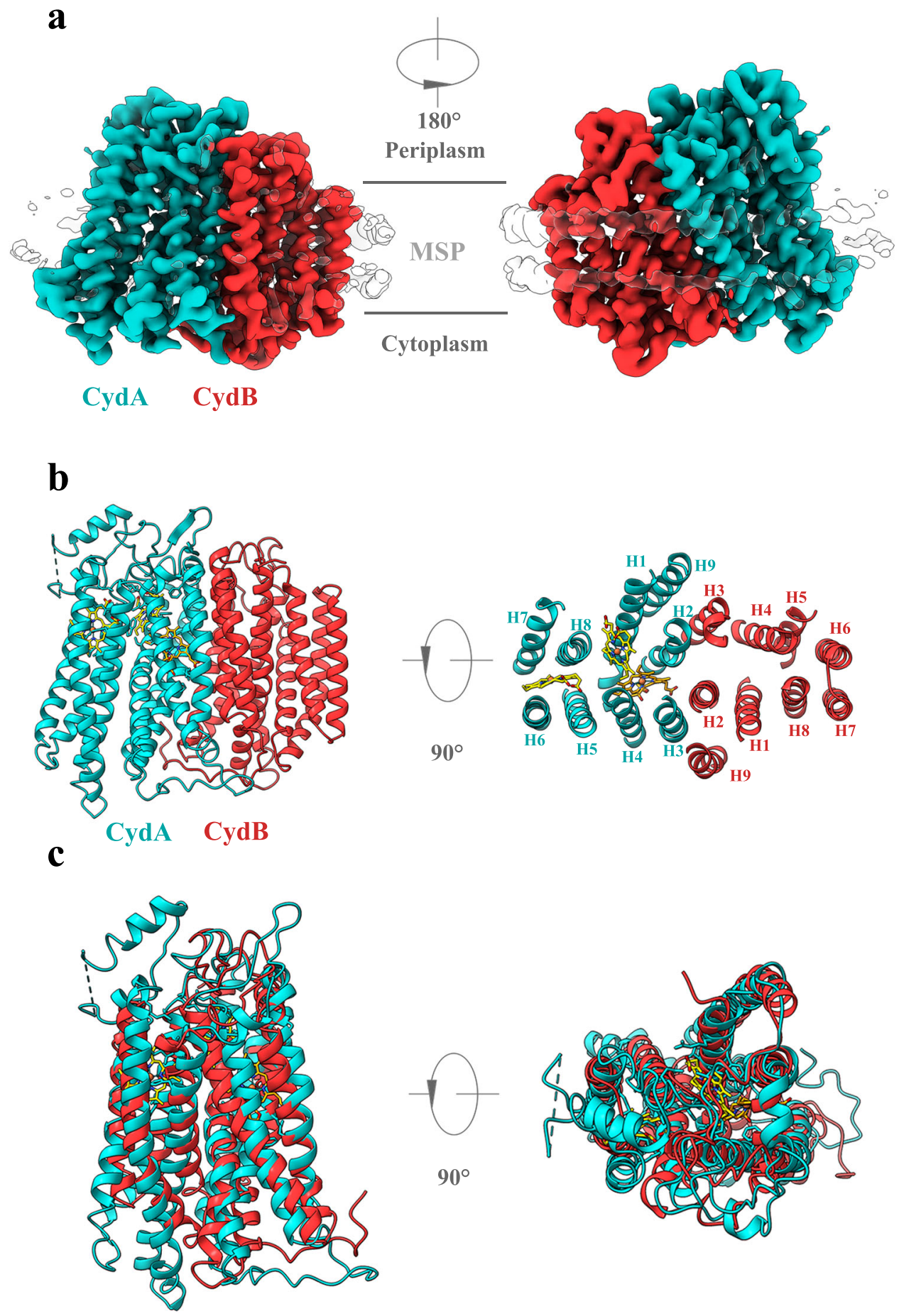

Fig. 1 Overall structure of the $\mathbf{M s m}$ bd oxidase. a The $b d$ oxidase cryo-EM density map at $2.8 \AA$ resolution. MSP, membrane scaffold protein. $\mathbf{b}$ Cartoon representation of the bd oxidase consisting of subunits CydA (red) and CydB (cyan). c Structural superposition of CydA and CydB.

enzymes $^{19-21}$ (Fig. 2b). It is noteworthy that the $\mathrm{Q}_{\mathrm{C}}$-loop here adopts a rigid secondary structure with a horizontal Qh2 (residues 317-327), that emerges from the flexible $\mathrm{Q}_{\mathrm{N}}$-loop part and covers the periplasmic surface of CydA. Site-directed mutagenesis and whole-bacteria assays, performed on the Mycobacterium tuberculosis (Mtb) bd oxidase, demonstrated that the regions corresponding to the $\mathrm{Q} h 2$ and the equivalent residues $\mathrm{Tyr}^{323}, \mathrm{Phe}^{327}$, and $\mathrm{Tyr}^{332}$ nearby the $\mathrm{Q} h 2$ stretch are essential for the function of the oxidase ${ }^{25}$ (Supplementary Fig. 8). In Msm bd oxidase, we observe that these three residues are involved in the interactions with Qh1 and the periplasmic loop between TMH 8/
9 , located at the periplasmic surface of heme groups $b_{558}$ and $b_{595}$, and as a result potentially affect the cofactor and quinol binding (Fig. 2a). Tyr ${ }^{323}$ forms the van der Waals interaction with Pro $^{258}$ in the Qh1 region, and $\mathrm{Phe}^{327}$ and $\mathrm{Tyr}^{332}$ form the stacking interactions with $\operatorname{Trp}^{404}$ in the loop TM8/9 region. So the observed structural features are in agreement with the previous functional characterization of the $M t b$ enzyme $^{25}$. Additionally, according to the structural superposition between $M s m$ and $E$. coli CydA subunits (Supplementary Fig. 6), the corresponding residues of $\mathrm{Tyr}^{323}, \mathrm{Ph}^{327}$, and $\mathrm{Tyr}^{332}$ in $\mathrm{Msm}$ are $\mathrm{His}^{314}, \mathrm{Tyr}^{353}$, $\mathrm{Ser}^{379}$ in E. coli, respectively. $\mathrm{His}^{314}$ and $\mathrm{Tyr}^{353}$ also form van der 


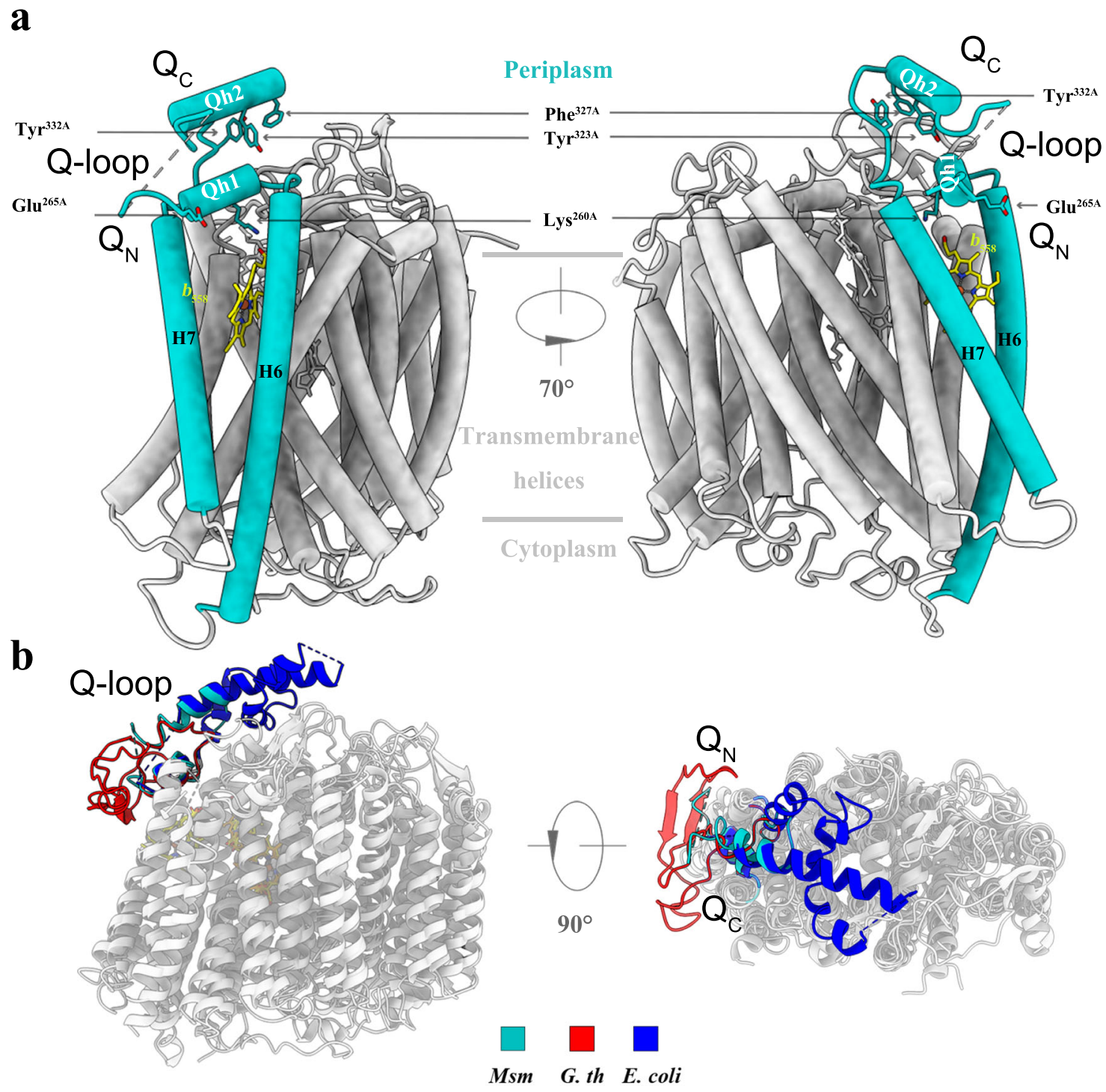

Fig. 2 The Q-loop in bd oxidases. In CydA, a hydrophilic region between the transmembrane helices 6 and 7 harbors the quinol-binding site and has thus been named the Q-loop. a The N-terminal and C-terminal Q loops are rigid and well-ordered helical segments, but the linking region between them is not resolved in the maps. The residues labeled are important for stability or activity. $\mathbf{b}$ The bd oxidases from $G$. th, E. coli, and Msm are superimposed. The Qloop domains from G. th, E. coli, and Msm are shown in red, blue, and cyan, respectively.

Waals interactions and a stacking interaction, respectively, with $\mathrm{Thr}^{251}$ in the Qh1 region and Trp ${ }^{451}$ in the loop TM8/9 region. Hence, these interactions in the Msm bd oxidase are very similar to those observed in the E. coli complex. It is also worth noting that the folding of the $\mathrm{Q}_{\mathrm{C}}$-loop region is different (Fig. 2b), compared to the other $b d$ oxidases, though the Q-loop domain of Msm bd oxidase should be remembered these belong to the short Q-loop class (Supplementary Fig. 9) ${ }^{20}$. This array of differences suggests that the Q-loop domain could be used as a marker for evolutionary analysis of $b d$ oxidases in prokaryotes (Supplementary Fig. 10).

Electron transfer in $\mathbf{M s m} \boldsymbol{b} \boldsymbol{d}$ oxidase. The three heme groups $\left(b_{558}, b_{595}\right.$, and $\left.d\right)$ unambiguously identified in $M s m$ bd oxidase are organized in a triangulated arrangement near the periplasmic side of CydA (Fig. 3a). In this structure, the low-spin $b_{558}$ is within the transmembrane core of subunit CydA, adjacent to the $\mathrm{Q}_{\mathrm{N}}$-loop segment. Its axial ligands are conserved residues His ${ }^{185}$ and $\mathrm{Met}^{346}\left(\mathrm{His}^{186}\right.$ and $\mathrm{Met}^{325}$ in G. th; His ${ }^{186}$ and $\mathrm{Met}^{393}$ in $E$. coli) ${ }^{19-21}$ (Fig. 3b). Heme $b_{595}$ is located closer to the periplasmic side and is ligated by $\mathrm{Glu}^{398}\left(\mathrm{Glu}^{378}\right.$ in $G$. th; Glu ${ }^{445}$ in $E$. coli) ${ }^{19-21}$ (Fig. 3b). There is a conserved $\operatorname{Trp}^{394 \mathrm{~A}}$ between heme groups $b_{595}$ and $b_{558}$ that may mediate electron transfer ${ }^{19-21,30}$. The third cofactor heme $d$, the site of oxygen binding and reduction, is positioned at the center of $\mathrm{CydA}$ and the invariant His $^{18}$ (His ${ }^{21}$ in G. th; His ${ }^{19}$ in E. coli) appears to be ligated to the heme iron on one side ${ }^{19-21}$

Although the $b d$ oxidases of $G$. th and $M s m$ are from the same S-subfamily, the relative arrangement of the three heme groups are strikingly different ${ }^{19}$ (Fig. 3c). The organization of the redox center in Msm cytochrome $b d$ is similar to that reported for the L-subfamily as exemplified by $E$. coli $b d$ oxidase $^{20,21}$. A structural superimposition (Fig. 3c) shows the two heme groups are located in the same position in the CydA, the distances between the central iron atoms are consistent as the orientations of the heme planes relative to the membrane plane. Therefore, the cofactor location is a conserved feature of the three respiratory $b d$ oxidases. On the opposite side of the heme $d, \mathrm{Glu}^{98}\left(\mathrm{Glu}^{101}\right.$ in G. $t h$; Glu ${ }^{99}$ in E. coli) acts as the axial 


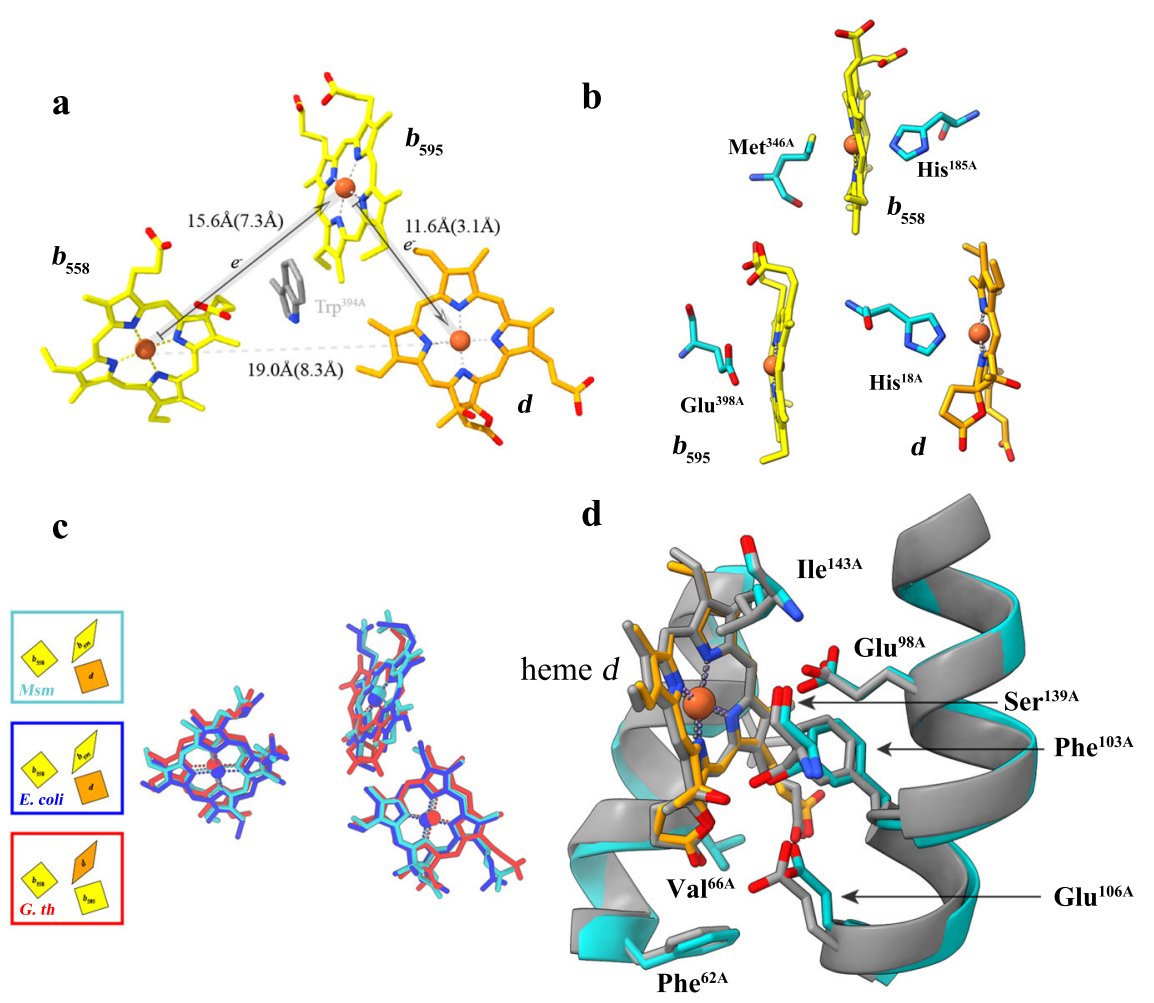

Fig. 3 Cofactor organization in $\mathbf{M s m}$ cytochrome bd. a Triangular arrangement of the heme cofactors in CydA. Heme edge-to-edge distances are indicated by numbers in the parentheses. b Axial amino acid ligands of the heme cofactors. c Heme superposition between E. coli, G. th, and Msm. d The superimposed heme $d$ sites from Msm and $E$. coli.

ligand. Glu 98 here is $6.2 \AA$ from the central iron atom. The equivalent distances in $G$. th and $E$. coli are 2.1 and $6.0 \AA$, respectively ${ }^{19-21}$. This voluminous cavity is suggested to be used for the binding of substrates such as oxygen, roofed by the hydrophobic Ile ${ }^{143}$ and Phe $\mathrm{P}^{103}$ (Ile $^{144}$ and Phe $\mathrm{Ph}^{104}$ in E.coli) ${ }^{20,21}$. The location and surrounding environment of the cofactors in the $b d$ oxidase are highly conserved between $M s m$ and E. coli (Fig. 3d). Collectively, it is suggested that a sequential electron transfer from heme $b_{558}$ via heme $b_{595}$ to heme $d$ also exists in the mycobacterial $b d$ oxidases ${ }^{20,21}$.

Two oxygen access channels in $\mathbf{M s m} \boldsymbol{b} \boldsymbol{d}$ oxidase. Cytochromes $b d$ play a role in energy metabolism with high $\mathrm{O}_{2}$ affinity under hypoxic conditions ${ }^{4,12}$, conditions often encountered by the microorganisms in their natural habitats. The dioxygen has to bind to heme $d$ and then is reduced at this position ${ }^{19-21}$. In $E$. coli $b d$ oxidase, the $\mathrm{O}_{2}$-channel acts as a pathway for direct oxygen diffusion from the membrane interior to the heme $d$ reaction site. It is formed by a small direct hydrophobic channel, which starts above $\operatorname{Trp}^{63}$ (Trp ${ }^{67}$ in $\left.M s m\right)$ at the membrane interface between TMH1 and TMH9 of CydB and extends further to heme $d$ on $\mathrm{CydA}^{20,21}$. The corresponding residue Trp has been demonstrated to be essential for $b d$ activity in $M t b^{25}$. Noteworthy, in the Msm structure, the channel is also formed by a conserved structural topology and residues according to a comparison between the $b d$ oxidases from E. coli and Msm (Supplementary Fig. 11), which suggests that the oxygen here may also access the active site through this conserved $\mathrm{O}_{2}$-channel (identified as channel 1) (Fig. 4). Intriguingly, there is an additional accessible channel directly connecting to the protein surface and extending to the heme $b_{595}$, which is also identified in the G. th enzyme $\mathrm{e}^{19}$. This channel has also been proposed for the oxygen entry site in
G. the bd oxidase ${ }^{19,20}$, which is blocked by the singletransmembrane subunit $\mathrm{CydH}$ in the $E$. coli enzyme ${ }^{20,21}$. In addition, the heme $d$ in this structure is buried deeper inside the subunit CydA and the penetration of dioxygen from this cavity into heme $d$ is also blocked by heme $d$ itself $^{21}$. However, the channel is accessible in the Msm enzyme and previous studies have reported that the high-spin heme $b_{595}$ could be the second reaction site for $\mathrm{O}_{2}{ }^{5,31}$. Therefore, this channel (channel 2) is very likely to be an alternative pathway to guide dioxygen to heme $b_{595}$, which may further sustain energy metabolism in the bacterial cell and enhance mycobacterial survivability in the host. It has been reported that the threshold $\mathrm{pO}_{2}\left(\mathrm{O}_{2}\right.$ tension) of the growth medium for the induction of cyd gene cluster in $M$. smegmatis (ca. $1 \%$ air saturation) ${ }^{32}$ is significantly lower than that of $E$. coli $(10 \% \text { air saturation })^{33}$, suggesting that the $M s m$ bd oxidase has a different functional or kinetic range with respect to oxygen availability compared to that of $E$. coli ${ }^{32}$. Overall, therefore, the two oxygen channels in $M s m b d$ oxidase are reasonably proposed based on these structural features and the previously described studies. Future investigations are needed to determine whether these two catalytic reactions take place at the same time.

Although the $b d$ oxidases do not pump protons from the cytoplasmic side to periplasmic side, producing the proton motive force across the membrane, the pathway for proton uptake from the cytoplasm is crucial to reduce dioxygen to water ${ }^{4}$. Two proton pathways in subunits CydA and CydB from the cytoplasmic side to the active site have been proposed in $G$. $t h^{19}$ and E. coli enzymes ${ }^{20,21}$. These studies indicate two hydrophilic channels for proton transfer. Based on the superimposition and structural analysis between the $b d$ oxidases from $G$. th and $E$. coli ${ }^{19,20}$, the relatively conserved hydrophilic residues in our model, along the canonical CydA and CydB pathways, are His ${ }^{125 . A}, \mathrm{Gln}^{36 . A}$, Glu ${ }^{106 . A}, S^{107 . A}, S^{139 . A}$ to Glu ${ }^{98 . A}$, and Asp ${ }^{25 . B}, A^{62 . B}$, and 


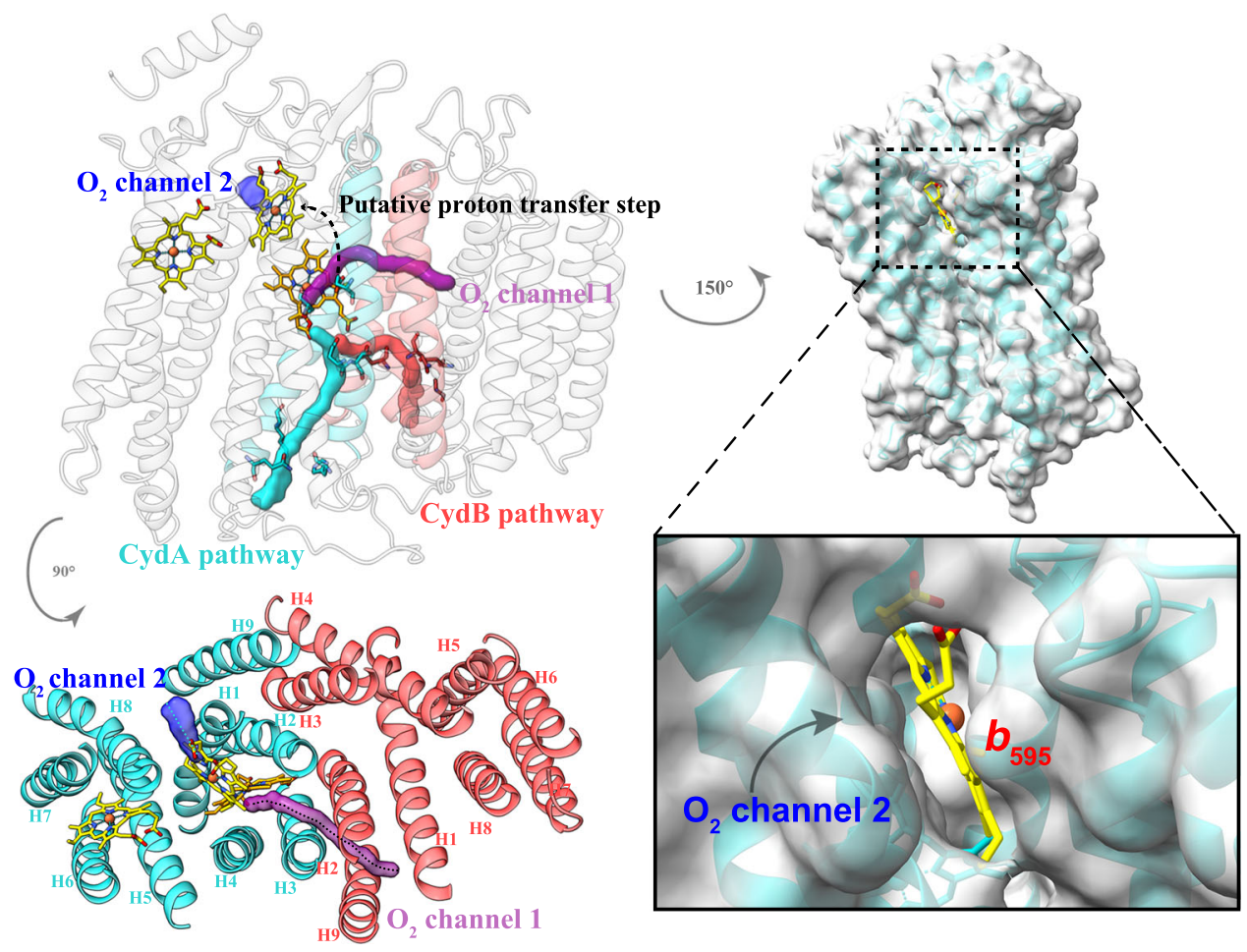

Fig. 4 Proton/gaseous substrate channels in the $\mathbf{M s m}$ bd oxidase. Subunits CydA/B are shown in cartoon representation. The putative dioxygen and proton channels are labeled. The heme groups are shown as stick models. The proton transfer step from heme $b_{595}$ to heme $d$ is identified by a black dashed path.

Asn $64 . B$ (Fig. 4, Supplementary Fig. 11). Given the conserved identity of the proton pathways in mycobacterial enzymes, they are also likely to facilitate proton transfer for dioxygen reduction at the heme $d$ site. In addition, in terms of the dioxygen reduction at the heme $b_{595}$ site, there must be an additional proton transfer step from heme $d$ to heme $b_{595}$ in order to deliver protons to the oxygen reduction site (Fig. 4 ), which may be potentially similar to that of the G. th enzyme ${ }^{19}$. According to the current structure, the heme propionate of heme $d$ is in a hydrophobic environment without any charge compensation. It is thus very likely protonated and supplies protons for unresolved water molecules here that connect heme $b_{595}$ to heme $d$. These protons would be replenished via the CydA/B proton pathways.

Overall, the electron released from the quinol bound at the quinone-binding site is transferred, in turn, to the prosthetic groups heme $b_{585}$, heme $b_{595}$ to heme $d$. At the same time, the oxygen molecule that is diffused to the heme $b_{595}$ and/or heme $d$ sites is reduced to water, a process that is involved in conducting protons to the oxygen-binding site through the CydA/B pathways (Fig. 5).

Cytochromes $b d$ is ubiquitous among prokaryotes (but not present in eukaryotes) and is now attracting attention as promising targets for next-generation antibacterials. Here, we have determined the $2.8 \AA$ cryo-EM structure of $M s m b d$ oxidase. The overall fold is similar to the two other previously reported $b d$ oxidases but exhibits several different features, including the fold of the Q-loop and the number of associated subunits. In addition, we have identified two potential oxygen access channels that look to be also present in $G$. th and $E$. coli cytochromes $b d$. The quinolbinding site located in the Q-loop has been proposed to be a target for drug discovery. However, the structure of the Q-loop has not been fully determined, thus posing a challenge for the design of quinol-type inhibitors. The two oxygen-conducting $\mathrm{O}_{2^{-}}$ channels could be alternative targets for the discovery of antituberculosis drugs.

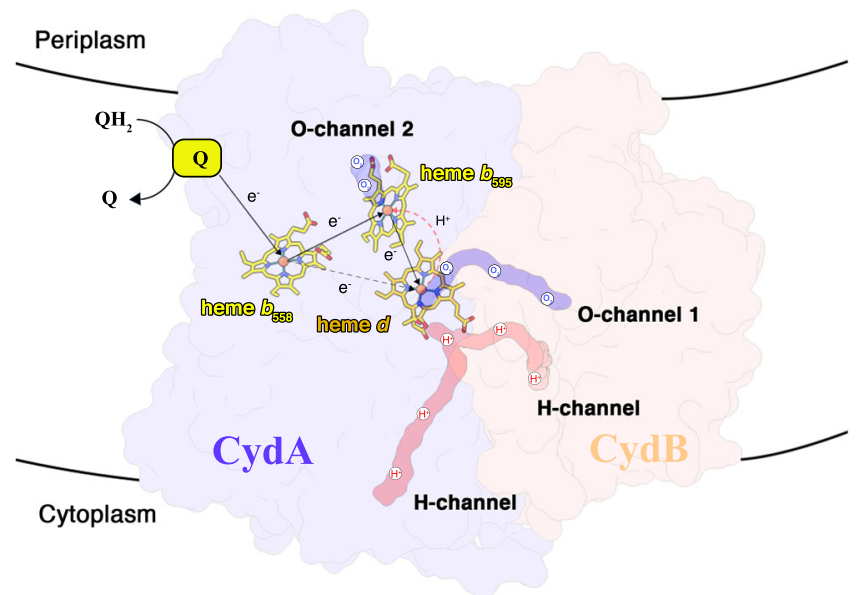

Fig. 5 A schematic diagram showing the electron/proton transfer pathway in Msm bd oxidase and the relevant oxygen entry pathway. The heme groups are shown as in stick models. The putative dioxygen and proton channels are labeled. Electron transfer directions are shown in black arrows.

\section{Methods}

Bacteria strain and culture. The $c y d A B$ gene was cloned into pMV261 plasmid with a 10x His tag at the C-terminus of $c y d B$. The primers are listed in Supplementary Table 2. Expression was achieved by electroporation of the plasmid into strain $\mathrm{Msm} \mathrm{mc}^{2} 51^{34}$. A volume of $1 \mathrm{~mL}$ strain stock was added to $24 \mathrm{~mL} \mathrm{LB}$ broth $(0.1 \% \mathrm{Tw} 80,50 \mu \mathrm{g} / \mathrm{mL}$ kanamycin, and $20 \mu \mathrm{g} / \mathrm{mL}$ carbenicillin) and cultured overnight at $37^{\circ} \mathrm{C}$ and $220 \mathrm{rpm}$. Next, $4 \mathrm{~mL}$ pre-culture aliquots were transferred to $1 \mathrm{~L}$ LB broth (rubber plug, $0.1 \%$ Tw $80,50 \mu \mathrm{g} / \mathrm{mL}$ kanamycin, and $20 \mu \mathrm{g} / \mathrm{mL}$ carbenicillin) and cultured at $37^{\circ} \mathrm{C}$ and $220 \mathrm{rpm}$. When the $\mathrm{OD}_{600}$ reached $0.8,5 \mathrm{~mL}$ of $40 \%$ acetamide was added to induce the expression of the target protein over 3 days at $25^{\circ} \mathrm{C}$ and $220 \mathrm{rpm}$

Protein purification and characterization. The purification procedure followed a previous study but with a few modifications ${ }^{30}$. Membranes of the cells were 
extracted in buffer (20 mM HEPES, $\mathrm{pH} 7.4,100 \mathrm{mM} \mathrm{NaCl}$ ), and then stirred slowly at $4{ }^{\circ} \mathrm{C}$ for $2 \mathrm{~h}$ with $1 \%(\mathrm{w} / \mathrm{v})$ dodecyl-beta-d-maltoside (DDM). The supernatant after centrifugation was loaded onto a Ni-NTA column and the eluted fraction including the protein of interest was loaded onto a Superdex 200 (GE Healthcare) column equilibrated in a buffer containing $20 \mathrm{mM}$ HEPES, $\mathrm{pH} 7.4,100 \mathrm{mM} \mathrm{NaCl}$, and $0.02 \%(\mathrm{w} / \mathrm{v})$ DDM. The peak fractions were analyzed by SDS-PAGE (sodium dodecyl sulfate-polyacrylamide gel electrophoresis), then pooled and concentrated to $6 \mathrm{mg} / \mathrm{mL}$.

Preparation of reduced quinol substrate. 2,3-Dimethyl-1,4-naphthoquinone (DMNQ, CAS 2197-57-1) was synthesized by WuXi AppTec. DMNQ reduction was performed following previously published protocols with some modifications ${ }^{35}$. To prepare the reduced quinol, $\mathrm{DMNQH}_{2}, 20 \mathrm{mM}$ DMNQ was ultrasonically dissolved in $1 \mathrm{~mL}$ ethanol with $6 \mathrm{mM} \mathrm{HCl}$. A few grains of sodium borohydride $\left(\mathrm{NaBH}_{4}\right)$ were then added to obtain a fully reduced, colorless solution in the ice-bath. An appropriate amount of $\mathrm{HCl}$ was used to quench the mixture under the protection of argon. The quinol solution was stored at $-80^{\circ} \mathrm{C}$.

Oxygen consumption assay. Oxidase activity was determined according to the previous studies ${ }^{20,36,37}$. Oxygen consumption was monitored with a Clark-type oxygen electrode (Hansatech Chlorolab 2) in the buffer 20 mM HEPES, pH 7.4, $100 \mathrm{mM} \mathrm{NaCl}, 0.04 \% \mathrm{DDM}$, and $10 \mathrm{mM}$ DTT at room temperature. To begin the assay, $480 \mu \mathrm{L}$ buffer was first added until the oxygen equilibrium. $20 \mu \mathrm{L} \mathrm{DMNQH}_{2}$ was then added and the substrate autoxidation rate was recorded. The reaction was started by the addition of $0.8 \mu \mathrm{M} b d$ complex. The time course for oxygen consumption was curved with GraphPad prime 6.0 software, from which an estimate of the observed pseudo-first-order rate constant $\left(k_{\mathrm{obs}}\right)$ is obtained (corrected for autoxidation). This assay included four groups of parallel experiments.

Reconstruction of cytochrome bd into nanodiscs. MSP1D1 was used to reconstruct the nanodisc and the purification and reconstruction followed the reported study $^{38}$. Briefly, cytochrome $b d$, MSPD1, and POPC were mixed with a stoichiometry of 1:4:160 and incubated at $4^{\circ} \mathrm{C}$ for one hour. Next, $200 \mu \mathrm{L}$ of resuspended Bio-Beads $(0.5 \mathrm{~g} / \mathrm{mL})$ were added twice with an interval of $30 \mathrm{~min}$ to remove the detergents. After $12 \mathrm{~h}$ of incubation, the supernatant was applied to a Superdex 200 (GE Healthcare) column equilibrated in $20 \mathrm{mM}$ HEPES, pH 7.4, $100 \mathrm{mM} \mathrm{NaCl}$ buffer. The peak fraction was collected and concentrated.

Cryo-EM sample preparation and data collection. Aliquots $(4 \mu \mathrm{L})$ of reconstructed nanodisc-cytochrome bd at a concentration of $1 \mathrm{mg} / \mathrm{mL}$ were applied to glow-discharged Quantifol $\mathrm{Cu}$ 1.2/1.3 (mesh 300) grids. For cytochrome bd and aurachin D complex, $0.35 \mathrm{mM}$ aurachin $\mathrm{D}$ was added and incubated with nanodisc-cytochrome bd for half an hour before sample vitrification. Glow discharge was accomplished by adding an $\mathrm{H}_{2}$ and $\mathrm{O}_{2}$ mixture in the Gatan Solarus 950 for $25 \mathrm{~s}$. After blotting for $3 \mathrm{~s}$ with a blot force of -2 , grids were flash-frozen in liquid ethane cooled by liquid nitrogen using an FEI Vitrobot operated at $8{ }^{\circ} \mathrm{C}$ and $100 \%$ humidity. For cytochome bd complex without aurachin D, data collection was achieved using the Titan Krios electron microscopy operated at $300 \mathrm{kV}$ with a Gatan K3 detector at a magnification of SA $\times 29,000$. Images were recorded in super-resolution mode binned to a pixel size of $0.82 \AA /$ pixel. Data acquisition was achieved by using serialEM ${ }^{39}$. Images were collected with 40 frames and a total dose of $60 \mathrm{e}^{-} / \AA^{2}$. The defocus range was set to $1.2-1.8 \mu \mathrm{m}$. For cytochrome bd-aurachin D complex, images were recorded using an FEI Titan Krios electron microscopy operating at $300 \mathrm{kV}$ with a Gatan $\mathrm{K} 2$ detector at a magnification of $\times 165,000$ with an energy filter, corresponding to a pixel size of $0.82 \AA /$ pixel. Images were collected with 40 frames and a total dose of $60 \mathrm{e}^{-} / \AA^{2}$ with a defocus range between 1.2 and $1.8 \mu \mathrm{m}$.

Image processing. Dose-fractioned images were motion-corrected and doseweighted by MotionCor2 software $^{40}$. CTF estimation was performed by cryoSPARC ${ }^{41}$. 1,578,284 particles were automatically picked and extracted with a box size of 256 pixels $^{41}$. 2D classification and 3D classification and refinement were all performed in cryoSPARC ${ }^{41} .50,000$ particles were used to generate three classes in ab-initio reconstruction. The classes were used as templates for heterogeneous refinement with all selected particles. After a few rounds of heterorefinement, 270,938 particles converged into one class with a $3.4 \AA$ initial map. These particles were used to perform a homogeneous refinement and local refinement to obtain a final resolution of $2.79 \AA$.

Model building and refinement. The final map was sharpened automatically using a $B$-factor of $121.3 \AA^{2}$ in cryoSPARC. The atomic model was manually built in $\operatorname{Coot}^{42}$ (version 0.8.9.1) using the crystal structure of G.th cytochrome bd (PDB: $5 \mathrm{doq})^{19}$ as a template. Real-space refinement and validation of the final model were performed in Phenix (version 1.14) ${ }^{43}$. The local resolution map was calculated with ResMap ${ }^{44}$. All reported resolutions were based on the gold-standard FSC 0.143 criteria ${ }^{45}$. $\mathrm{FSC}_{\text {work }}$ and $\mathrm{FSC}_{\text {test }}$ were conducted to check for over fitting ${ }^{46}$.

All figures were created using UCSF Chimera ${ }^{47}$ or $\mathrm{PyMOL}^{48}$.
Reporting summary. Further information on research design is available in the Nature Research Reporting Summary linked to this article.

\section{Data availability}

The accession numbers for the 3D cryo-EM density map of $M s m b d$ oxidase without and with bound AD in present study are EMD-30582 and EMD-31302, respectively. The accession number for the coordinates for the Msm bd oxidase without bound $\mathrm{AD}$ in this study is PDB: 7D5I. Source data are provided with this paper.

Received: 16 November 2020; Accepted: 14 July 2021; Published online: 30 July 2021

\section{References}

1. Michel, H. Terminal oxidases of the heme-copper and bd oxidase type, a structural and functional comparison. Biochim. Biophys. Acta 1859, e3 (2018).

2. Borisov, V. B. \& Siletsky, S. A. Features of organization and mechanism of catalysis of two families of terminal oxidases: heme-copper and bd-type. Biochemistry 84, 1390-1402 (2019).

3. Mitchell, P. Coupling of phosphorylation to electron and hydrogen transfer by a chemiosmotic type of mechanism. Nature 191, 144-148 (1961).

4. Borisov, V. B. et al. The cytochrome bd respiratory oxygen reductases. Biochim. Biophys. Acta 1807, 1398-1413 (2011).

5. D'Mello, R., Hill, S. \& Poole, R. K. The cytochrome bd quinol oxidase in Escherichia coli has an extremely high oxygen affinity and two oxygen-binding haems: implications for regulation of activity in vivo by oxygen inhibition. Microbiology 142, 755-763 (1996).

6. Belevich, I. et al. Cytochrome bd from Azotobacter vinelandii: evidence for high-affinity oxygen binding. Biochemistry 46, 11177-11184 (2007).

7. Baughn, A. D. \& Malamy, M. H. The strict anaerobe Bacteroides fragilis grows in and benefits from nanomolar concentrations of oxygen. Nature 427, 441-444 (2004).

8. Shi, L. et al. Changes in energy metabolism of Mycobacterium tuberculosis in mouse lung and under in vitro conditions affecting aerobic respiration. Proc. Natl Acad. Sci. USA 102, 15629-15634 (2005).

9. Mason, M. G. et al. Cytochrome bd confers nitric oxide resistance to Escherichia coli. Nat. Chem. Biol. 5, 94-96 (2009).

10. Forte, E. et al. Cytochrome bd oxidase and hydrogen peroxide resistance in Mycobacterium tuberculosis. mBio 4, e01006-e01013 (2013).

11. Huycke, M. M. et al. Extracellular superoxide production by Enterococcus faecalis requires demethylmenaquinone and is attenuated by functional terminal quinol oxidases. Mol. Microbiol. 42, 729-740 (2001).

12. Mascolo, L. \& Bald, D. Cytochrome bd in Mycobacterium tuberculosis: a respiratory chain protein involved in the defense against antibacterials. Prog. Biophys. Mol. Biol. 152, 55-63 (2020).

13. Kalia, N. P. et al. Exploiting the synthetic lethality between terminal respiratory oxidases to kill Mycobacterium tuberculosis and clear host infection. Proc. Natl Acad. Sci. USA 114, 7426-7431 (2017).

14. Endley, S., McMurray, D. \& Ficht, T. A. Interruption of the cydB locus in Brucella abortus attenuates intracellular survival and virulence in the mouse model of infection. J. Bacteriol. 183, 2454-2462 (2001).

15. Duc, K. M. et al. The small protein CydX is required for Cytochrome bd quinol oxidase stability and function in Salmonella enterica Serovar Typhimurium: a Phenotypic Study. J. Bacteriol. 202, e00348-19 (2020)

16. Wilson, R. P. et al. STAT2 dependent Type I Interferon response promotes dysbiosis and luminal expansion of the enteric pathogen Salmonella Typhimurium. PLoS Pathog. 15, e1007745 (2019).

17. Corbett, D. et al. Listeria monocytogenes has both Cytochrome bd-type and Cytochrome aa (3)-type terminal oxidases, which allow growth at different oxygen levels, and both are important in infection. Infect. Immun. 85, e00354-17 (2017)

18. Lee, B. S., Sviriaeva, E. \& Pethe, K. Targeting the cytochrome oxidases for drug development in mycobacteria. Prog. Biophys. Mol. Biol. 152, 45-54 (2020)

19. Safarian, S. et al. Structure of a bd oxidase indicates similar mechanisms for membrane-integrated oxygen reductases. Science 352, 583-586 (2016).

20. Safarian, S. et al. Active site rearrangement and structural divergence in prokaryotic respiratory oxidases. Science 366, 100-104 (2019)

21. Theßeling, A. et al. Homologous bd oxidases share the same architecture but differ in mechanism. Nat. Commun. 10, 5138 (2019).

22. Goojani, H. G. et al. The carboxy-terminal insert in the Q-loop is needed for functionality of Escherichia coli cytochrome bd-I. Biochim. Biophys. Acta Bioenerg. 1861, 148175 (2020).

23. Theßeling, A. et al. The long Q-loop of Escherichia coli cytochrome bd oxidase is required for assembly and structural integrity. FEBS Lett. 594, 1577-1585 (2020). 
24. Harikishore, A. et al. Targeting the menaquinol binding loop of mycobacterial cytochrome bd oxidase. Mol. Divers. 25, 517-524 (2021).

25. Sviriaeva, E. et al. Features and functional importance of key residues of the Mycobacterium tuberculosis Cytochrome bd oxidase. ACS Infect. Dis. 6, 1697-1707 (2020).

26. Hoeser, J. et al. Subunit CydX of Escherichia coli cytochrome bd ubiquinol oxidase is essential for assembly and stability of the di-heme active site. FEBS Lett. 588, 1537-1541 (2014).

27. Matsumoto, Y. et al. Mass spectrometric analysis of the ubiquinol-binding site in cytochrome bd from Escherichia coli. J. Biol. Chem. 281, 1905-1912 (2006).

28. Mogi, T. et al. Probing the ubiquinol-binding site in cytochrome bd by sitedirected mutagenesis. Biochemistry 45, 7924-7930 (2006).

29. Lu, P. et al. The anti-mycobacterial activity of the cytochrome bcc inhibitor Q203 can be enhanced by small-molecule inhibition of cytochrome bd. Sci. Rep. 8, 2625 (2018).

30. Gong, $\mathrm{H}$. et al. An electron transfer path connects subunits of a mycobacterial respiratory supercomplex. Science 362, eaat8923 (2018).

31. Rothery, R. A., Houston, A. M. \& Ingledew, W. J. The respiratory chain of anaerobically grown Escherichia coli: reactions with nitrite and oxygen. J. Gen. Microbiol. 133, 3247-3255 (1987).

32. Kana, B. D. et al. Characterization of the cydAB-encoded cytochrome bd oxidase from Mycobacterium smegmatis. J. Bacteriol. 183, 7076-7086 (2001).

33. Becker, $\mathrm{S}$. et al. Regulatory $\mathrm{O} 2$ tensions for the synthesis of fermentation products in Escherichia coli and relation to aerobic respiration. Arch. Microbiol. 168, 290-296 (1997).

34. Li, X. et al. Draft Genome sequence of mc2 51, a highly hydrogen peroxideresistant Mycobacterium smegmatis mutant strain. Genome Announc. 2, e00092-14 (2014).

35. Bennett, M. C. et al. Chronic in vivo sodium azide infusion induces selective and stable inhibition of cytochrome c oxidase. J. Neurochem. 66, 2606-2611 (1996).

36. Bonner, W. D., Clarke, S. D. \& Rich, P. R. Partial purification and characterization of the quinol oxidase activity of Arum maculatum mitochondria. Plant Physiol. 80, 838-842 (1986).

37. Kusumoto, K. et al. Menaquinol oxidase activity and primary structure of cytochrome bd from the amino-acid fermenting bacterium Corynebacterium glutamicum. Arch. Microbiol. 173, 390-397 (2000).

38. Ritchie, T. K. et al. Chapter 11-reconstitution of membrane proteins in phospholipid bilayer nanodiscs. Methods Enzymol. 464, 211-231 (2009).

39. Mastronarde, D. N. Automated electron microscope tomography using robust prediction of specimen movements. J. Struct. Biol. 152, 36-51 (2005).

40. Zheng, S. Q. et al. MotionCor2: anisotropic correction of beam-induced motion for improved cryo-electron microscopy. Nat. Methods 14, 331-332 (2017).

41. Punjani, A. et al. cryoSPARC: algorithms for rapid unsupervised cryo-EM structure determination. Nat. Methods 14, 290-296 (2017).

42. Emsley, P. et al. Features and development of Coot. Acta Crystallogr. D 66, 486-501 (2010).

43. Adams, P. D. et al. Advances, interactions, and future developments in the CNS, Phenix, and Rosetta structural biology software systems. Annu. Rev. Biophys. 42, 265-287 (2013).

44. Kucukelbir, A., Sigworth, F. J. \& Tagare, H. D. Quantifying the local resolution of cryo-EM density maps. Nat. Methods 11, 63-65 (2014).

45. Rosenthal, P. B. \& Henderson, R. Optimal determination of particle orientation, absolute hand, and contrast loss in single-particle electron cryomicroscopy. J. Mol. Biol. 333, 721-745 (2003).

46. Brown, A. et al. Tools for macromolecular model building and refinement into electron cryo-microscopy reconstructions. Acta Crystallogr. D 71, 136-153 (2015).
47. Pettersen, E. F. et al. UCSF Chimera-a visualization system for exploratory research and analysis. J. Comput. Chem. 25, 1605-1612 (2004).

48. The PyMOL molecular graphics system, version 1.3r1. (Schrödinger, LLC. 2010).

\section{Acknowledgements}

We thank Dr. Chao Peng of the Mass Spectrometry System at the National Facility for Protein Science in Shanghai (NFPS), Zhangiiang Lab, SARI, China for data collection and analysis and Prof. Tongbu Lu and Yali Bai (School of Materials Science and Engineering, Tianjin University of Technology) for their technical support on Clark-type oxygen electrode and oxygen consumption assay. We would like to thank the Bio-Electron Microscopy Facility of ShanghaiTech University, and we are grateful to Dr. Qianqian Sun for her help with cryo-EM technical support. This work was supported by Grants from the National Key Research and Development Program of China (Grant No. 2017YFC0840300), the Strategic Priority Research Program of the Chinese Academy of Sciences (Grant No. XDB37030201, XDB37020203), and the National Natural Science Foundation of China (Grant Nos. 81520108019, 813300237).

\section{Author contributions}

Z.R. and H.G. conceived and supervised the study. W.W. purified the M. smegmatis bd complex. Y.T. and Y.L. performed activity identification of the purified M. smegmatis bd complex. W.W. and Y.G. collected and processed cryo-EM data and built the structure model; H.G., W.W., Y.G., Q.W., X.Z., Y.T., S.Z., Y.Z., Y.L., X.Y., F.L., L.W.G., and Z.R analyzed the structure and discussed the results and the manuscript was written by H.G., W.W., L.W.G. and Z.R.

\section{Competing interests}

The authors declare no competing interests.

\section{Additional information}

Supplementary information The online version contains supplementary material available at https://doi.org/10.1038/s41467-021-24924-w.

Correspondence and requests for materials should be addressed to Q.W., Z.R. or H.G.

Peer review information Nature Communications thanks Tim Rasmussen and the other, anonymous, reviewer(s) for their contribution to the peer review of this work.

Reprints and permission information is available at http://www.nature.com/reprints

Publisher's note Springer Nature remains neutral with regard to jurisdictional claims in published maps and institutional affiliations.

Open Access This article is licensed under a Creative Commons Attribution 4.0 International License, which permits use, sharing, adaptation, distribution and reproduction in any medium or format, as long as you give appropriate credit to the original author(s) and the source, provide a link to the Creative Commons license, and indicate if changes were made. The images or other third party material in this article are included in the article's Creative Commons license, unless indicated otherwise in a credit line to the material. If material is not included in the article's Creative Commons license and your intended use is not permitted by statutory regulation or exceeds the permitted use, you will need to obtain permission directly from the copyright holder. To view a copy of this license, visit http://creativecommons.org/ licenses/by/4.0/

(C) The Author(s) 2021 